\title{
An die Herren Mitarbeiter der Zeitschrift.
}

Bezugnebmend auf das 1887 erschienene Circular an die Leser und Mitarbeiter der Zeitschr. für Kryst. und Min., von welchem noch einzelne Exemplare auf Wunsch zur Verfügung stehen, erlauben sich die Unterzeichneten folgende Bestimmungen in geneigte Erinnerung zu bringen:

1) Arbeiten, deren wesentlicher Inhalt vorher oder gleicbzeitig in einer anderen deutschen Zeitschrift erscheint, sowie sogenannte "vorläufige Mitheilungen " sind principiell von der Aufnabme ausgeschlossen.

2) Das zu den Manuscripten benutzte Papier ist nur e in seitig zu beschreiben.

3) Die Manuscripte sind gut leserlich zu schreiben und auf die Anordnung von Tabellen, Formeln und dergleichen die grösstmögliche Sorgfalt zu verwenden (die erheblichen Correcturkosten, welche Aenderungen im Arrangement solcher Theile des Satzes erfordern, fallen, wenn sie durch Undeutlichkeit des Manuscriptes verursacht werden, ebenso wie diejenigen grösserer nachträglicher Einschallungen, dem Autor zur Last).

4) Die Zahl der gewünschten Separate, Notizen betreffend etwaiger Correclursendungen u. s. w. sind auf der ersten Seite des Manuscriptes einzutragen.

5) Alle Figuren sind auf besonderen Blättern beizulegen und zwar jede Figur a u einem besonderen Blatte.

Betreffs der Illustrationen gelten folgende Grundsätze :

a) Die Abbildung flächenreicher Krystalle von Mineralien, ferner mikroskopische und complicirtere Figuren werden lithographisch vervielfältigt. Die hierzu bestimmten Originalfiguren werden am besten nicht in Tusche, sondern in feiner B le istiftzeichnung auf glattem Papiere geliefert, und können auf demselben (da sie unter sachverständiger Anleitung vom Lithographen auf Stein copirt werden) auch die benutzten Hülfslinien stehen bleiben; letztere sind sogar erwünscht, um den Lithographen die Controle der Richtigkeit seiner Copie zu erleichtern; in den Fällen, wo die Verticale nicht durch Linien der Figur selbst repräsentirt wird, ist die Einzeichnung dieser Hülfslinien nothwendig zur richtigen Orientirung der Figur. Je sorgfältiger das eingesandte Original ausgeführt, je deutlicher die Richtung auch der kürzesten Kanten, etwa durch beigesetzte feine Hülfslinien, bezeichnet ist, auf desto bessere Wiedergabe hat der Autor zu rechnen. Derselbe schone also nicht die Mühe, etwa durch beigegebene Skizzen in grösserem Maassstabe und durch besondere Erläuterungen, undeutliche oder misslungene Stellen der Zeichnung unzweideutig zu erklären I Wenn durch Eintragung der Buchstaben oder sonstiger Zeichen, z. B. auf schraffirten Stellen, eine Figur undeutlich werden würde, so setze man die Buchstaben etc. neben dieselbe und bezeichne den Ort, wo jene eingetragen werden sollen, durch farbige Punkte oder punktirte Hinführungslinien mit deutlich markirten Endpunkten.

b) Als Textillustrationen werden gegeben die meist einfacheren Abbildungen der Krystallformen künstlich dargestellter Substanzen, sowie auch zu Arbeiten anderer Art gehörige Figuren, falls dieselben nicht nothwendig lithographische Wiedergabe erfordern und für sich eine Tafel nicht ausfüllen würden. 
Beck * $^{*}$ ) untersuchte 1877 die kobalthaltigen Varieläten Danaït und Glaukodot, um zu erfahren, inwieweit jene Variationen des Prismenwinkels in Beziehungen zu dem Kobaltgehalte standen. Diese Untersuchungen, sowie Sadebeck's gleich darauf in derselben Zeitschrift publicirten, gaben widersprechende Resultate, und können deswegen nicht in Betracht gezogen werden, weil die Zusammensetzung nur nach älteren Analysen angegeben wurde, während, wie ich im Folgenden zeigen will, die Constitution und Krystallform an jedem Fundorte ganz beträchtlich wechseln.

1878 lieferte Arzruni einen wichtigen Beitrag zur vorliegenden Frage, auf welche er einige Jahre später, zusammen mit Bärw ald, wieder zurückkam $\left.{ }^{* *}\right)$. Diese Forscher beschäftigten sich mit den Eisenarsenkiesen, von welchen neun Varietäten gemessen und analysirt wurden.

Zuerst wurde constatirt, dass die früher beobachtete Variation der Constanten in naher Beziehung zum Gehalte an Schwefel steht, insofern eine Vergrösserung der $a$-Axe um 0,00001 einem Zuwachse des Schwefelgehaltes von $0,00236 \%$ entspricht. Dagegen lässt sich diese Relation nicht auf die reine Schwefel- resp. Arsenikverbindung ubertragen, denn aus dem genannten Verhältnisse wird die $a$-Axe bei Markasit zu 0,7246, entsprechend einem Prisma von $71^{0} 51 \frac{1}{2}^{\prime}$, berechnet, während der wirkliche Werth $73^{\circ} 35^{\prime}$; bei Löllingit würde sich $61027 \frac{1}{2}$ berechnen, während $\mathrm{Schrauf} 66^{\circ} 41 \frac{1}{2}^{\prime}$ und B rögger $67^{\circ} 33 \frac{1}{2}^{\prime}$ beobachleten.

Ferner, und dies ist besonders bemerkenswerth, ging aus den Analysen hervor, dass die oben angefuhrte Formel $\mathrm{Fe}(S, A s)_{2}$ nicht der Zusammensetzung des Arsenkies entsprach, denn das Verhaltniss $F e:(S+A s)$ wich von 1: 2 so beträchtlich ab, dass dies nicht in Fehlern der Analyse begrundet werden konnte. Die von Arzruni und Bärwald ausgefuhrten Analysen beziehen sich auf acht verschiedene Arsenkiesvarietäten, deren $F e$ - und $S$-Gehalt bestimmt wurden. Von diesen lieferten vier Analysen Werthe, welche ziemlich gut der allgemein adoptirten Formel entsprachen, nämlich die Varietäten von Hohenstein, Freiberg, Binnenthal und der sogenannte „Plinian«. Die grösste Differenz bot der erste dar, wo $F e:(S+A s)=1$ : 1,963 anstatt 1: 2 war. Diese Differenz liegt jedoch innerhalb der Grenzen analytischer Fehler. Unter den übrigen waren zwei besonders bemerkenswerth. Das ausserste Extrem bildete das Mineral von Sala, von welchem $\frac{1}{3}$ g Substanz 39,96\% $\mathrm{Fe}$ und $20,40 \% \mathrm{~S}$ ergabea; hieraus folgt das Verhältniss $F e:(S+A s)=1: 1,83$. Am nächsten kommt diesem der Arsenkies. von Joachimsthal mit der Relation 1:1,86, von welchem etwa $\frac{1}{4} g$ analysirt wurde. Die Abweichung ist hier grösser, als dass sie ausschliesslich von der Analysenmethode herrühren könnte. Diese Thatsachen veranlassten

*) Tschermak's min.-petrogr. Mitth. 1877, S. 101. Ref. in dieser Zeitschr. 2, 518.

**) Diese Zeitschr. 2, 430; 7, 337. 
den genannten Forscher zu dem Schlusse, dass die Constitution der Arsenkiese die Deutung nichtzulasse, dass dieselben isomorphe Mischungen von $\mathrm{FeS}_{2}$ und $\mathrm{Fe} \mathrm{As}_{2}$ seien. Da bei keinem einzigen Fundorte die Zusammensetzung vollstiandig mit derjenigen eines anderen Fundortes ubereinstimmt, muss vielmehr jedes Arsenkiesvorkommen seine besondere, höchst complicirte Formel von dem Typus $\mathrm{Fe}_{2}+x \mathrm{Fe}_{m} A s_{n}$ besitzen.

Abgesehen von den Schwierigkeiten, welche dadurch bei der Erklärung der Relation zwischen der $a$-Axe und dem Schwefelgehalte entsteben, bietet auch eine Constitution, die dem Gesetze der multiplen Proportionen direct widerspricht, etwas so Interessantes dar, dass die Frage eine nochmalige Erörterung verdient.

Schon vor mehreren Jahren habe ich einige Untersuchungen uber diesen Gegenstand begonnen*), die sich auf Arsenkiese von W.-Silfberg, Dalarne, beziehen. Dabei zeigte es sich, dass an diesem Fundorte drei verschiedene Typen vorkommen. Der eine von diesen zeigte in der Zusammensetzung eine ganz bestimmte Abweichung von der Formel $\mathrm{Fe}(S, A s)_{2}$ in der von Arzruni nachgewiesenen Richtung. Zufälligerweise fertigte ich einige Dünnschliffe an von dem Gesteine, in welchem dieser Arsenkies vorkommt, und es zeigte sich dadurch, dass dies Mineral constant Einschlusse enthielt (was alsdann auch durch Reactionen leicht nachgewiesen werden konnte), welche jene Abweichung veranlassten. An Fundorten in derselben Gegend babe ich seitdem drei andere Typen gefunden, welche, nebst einigen früher bekannten Eisen- und Kobalt-Arsenkiesen einer kritischen Untersuchung unterzogen wurden, um Aufschluss über die Constitution der Arsenkiese zu erhalten.

Dabei war immer der Gesichtspunkt massgebend, dass nur solches Material für die Analyse zu benutzen war, welches ich vorher goniometrisch untersucht hatte, und in jedem Falle musste noch entschieden werden, ob und in welcher Weise die Krystalle von anderen Mineralien verunreinigt waren. Da die Untersuchung zeigte, dass der Arsenkies kaum ein einziges Mal rein zu finden ist, so hängt der Werth der Analysen gleich viel von der Bestimmung oder eventuell der Entfernung der fremden Mineralien, als von den Analysenmethoden ab.

Bei der Analyse war ich stets bemüht, solche Methoden zu benutzen, die zuverlässige Zahlen für alle Bestandtheile lieferten, was natürlich danu von besonderem Gewichte ist, wenn ihre gegenseitige Relation ermittelt werden soll. Wird dagegen - was nahe liegt, da oftmals nur drei Elemente zu bestimmen sind - der eine durch den Verlust der Analyse ermittelt, so wirkt eine zu hohe Zahl fur den èinen Stoff deprimirend auf die des anderen, und in der gegenseitigen Relation wird der Febler noch mehr ver-

*) Öfvers. af Kgl. Vetensk. Akad.-Förh. (Stockholm) 1888, No. 9. 
grössert werden. Zur Bestimmung des Eisens (und eventuell $\mathrm{Co}$ und $\mathrm{Ni}$ ) wurde das grob gepulverte Mineral in concentrirter Salpetersäure von etwa 1,40 spec. Gew. gelöst, anfangs ohne Erwärmen. Nach vorsichtigem Abdampfen zur Trockne wird in der Regel der abgeschiedene Schwefel oxydirt; der Rückstand wird in verdünnter Salpetersäure gelöst, das Arsen mit $\mathrm{H}_{2} \mathrm{~S}$ abgeschieden und als $\mathrm{Mg}$ - $\mathrm{Am}$-Arseniat bestimmt; aus dem $\mathrm{H}_{2} \mathrm{~S}$ enthaltenden Filtrate werden die Metalle auf gewöhnliche Weise bestimmt.

Die Salpetersäure-haltige Lösung ist, nach meiner Erfahrung, für die Schwefelbestimmung nicht gut brauchbar; auch wenn man die Salpetersäure durch Salzsäure verdrängt, ist es recht schwierig, die Schwefelsäure exact zu bestimmen. Besser gelingt folgende Methode, die bei Benutzung vollkommen reiner Reagentien gute Resultate liefert und sogar eine Controlbestimmung des Eisens zulässt. Das fein pulverisirte Mineral wird mit der zehnfachen Menge Kaliumnitrat und Natriumcarbonat geschmolzen. Ein Verlust von Schwefel ist bei vorsichtigem Erhitzen nicht zu befürchten, und wenn die Schmelze nach dem Erkalten in ammoniakalischem Wasser gelöst wird, so kann das Eisenoxyd meist klar filtrirt und ausgewaschen werden. Die Arsen und Schwefelsäure enthaltende Lösung wird mit $\mathrm{HCl}$ sauer gemacht, die Salpetersäure von jener Säure vollständig verdrängt und der Schwefel in gewöhnlicher Weise bestimmt. Enthält der Arsenkies Einschlüsse von fremden Mineralien, oder sind die Reagentien nicht absolut rein, so wird der Eisengehalt vergrössert, der also nach dieser Methode in der Regel etwas zu gross ausfällt.

\section{Wester - Silfberg, T y pus I.}

Die Gruben von Wester-Silfberg im Kirchspiel Norrbärke in Dalecarlien sind schon lange wegen ihrer Blei- und Silbererze bekannt. In allerletzter Zeil wurden sie auf Zinkblende belrieben, nachdem einige Jahre hindurch hauptsächlich manganhaltiges Eisenerz gewonnen worden war. In dem früher erwähnten Aufsatze, sowie in „Untersuchung schwedischer Mineralien (*), babe ich dieses Mineralvorkommen beschrieben. Die Erze finden sich als Einlagerungen in granulitischen Gebirgsarten, welche ausser aus Granulit hauptsächlich aus Quarzit- und Glimmerschiefer bestehen.

Obschon Arsenkies in mehreren Gruben vorkommt, habe ich nur in dem südlicben Theile der Grubenfelder Krystalle von diesem Minerale angetroffen. Die Felsart ist hier granatführender Glimmerschiefer, der oft in Quarzit ubergeht; in diesem liegen Stöcke von Zinkblende und Bleiglanz, Magnetkies u. a. Mineralien. Zuweilen traten auch Einlagerungen von Manganocalcit auf.

*) Tschermak's min.-petrogr. Mitth. 1885, S. 108. Ref. in dieser Zeitschr. 12, 90. 
Das Mineral kommt hier, sogar in derselben Grube, von verschiedener Ausbildung und Zusammensetzung, sowie in etwas verschiedener Gesellschaft vor, daher die Krystalle in drei Typen getheilt werden können. Der gewöhnlichste von diesen, der auch in mehrere Mineraliensammlungen ge langte, stimmt im Habitus und theilweise in den Constanten mit Breithaupt's von demselben Fundorte stammenden "Dalarnit (*) überein. Diese Krystalle, Typus I, liegen in einem Gemenge von Magnetkies, Kupferkies, Zinkblende, Bleiglanz, Schwefelkies, Magnetit, Kalkspath und Quarz, von welchen jedoch der erste sehr uberwiegt. Der Arsenkies, von dem jedes Individuum ringsum ausgebildet ist, kommt allein in Krystallen vor, und gehört entschieden zu den ersten Ausscheidungsproducten des Gemenges. Mit wenigen Ausnahmen scheinen die Krystalle ganz homogen zu sein, nur zuweilen umschliessen sie hie und da deutliche Bleiglanz- und Zinkblendekörner. Beim Zerschlagen solcher Stufen fallen gewöhnlich die Krystalle ganz heraus und liefern dabei negative Eindrucke, die an Handstücken gewöhnlich sind.

Die Krystalle besitzen eine ganz ungewöhnliche Grösse, so dass Individuen von $1 \mathrm{~cm}$ Länge und Breite ganz allgemein sind. Die meisten zeigen nur das Prisma $m\{110\}$ und das Brachydoma $l\{011\}$, die oftmals im Gleichgewicht sind (Fig. 1, Taf. I); bei prismatischer Ausbildung herrscht gewöhnlich das Prisma, selten das Doma vor. Die Kante zwischen (011) und (0T1) wird zuweilen durcb $s\{012\}$ zugeschärft (Fig. 2), diese' Flächen sind jedoch sebr schmal und fehlen oft. Keine anderen Flächen wurden an zahlreichen Individuen beobachtet.

Contactzwillinge nach $m\{110\}$ sind sehr selten; das eine Individuum ist in diesem Falle sehr klein und sitzt am Prisma des anderen. Das Mineral, welches nach $m\{110\}$ sehr undeutlich spaltet, hat eine ungewöhnlich dunkle Farbe, übereinstimmend mit metallischem Nickel, was wohl auf einem dünnen Ueberzuge von Magnetkies beruht. Der Glanz ist stark, besonders auf dem Prisma. An solchen Krystallen, welche längere Zeit auf den Halden gelegen hatten, sind die Flächen ganz matt, was von der Zersetzung des Magnetkies herrührt.

Ich habe am Goniometer eine grosse Anzahl Krystalle untersucht, führe jedoch hier nur diejenigen Winkelwerthe an, welche an vier Krystallen mit sehr scharfen Reflexen erhalten wurden.

*) Journ. f. pr. Chemie 1835, 4, 248. 


\begin{tabular}{|c|c|c|c|}
\hline Flächen: & Fund. -Winkel : & Grenzwerthe: & Ber. Winkel \\
\hline$m: m=(1 \mid 0):(1 \bar{T} 0)$ & $={ }^{*} 68^{0} 45^{\prime}$ & $68^{0} 32^{\prime}-68^{0} 50^{\prime}$ & - \\
\hline$s: s=(012):(0 T 2)$ & $=\quad-$ & $6114-6150$ & $61^{0} 33^{\prime}$ \\
\hline$: l=(012):(011)$ & $=-$ & $4-1923$ & $1912 \frac{1}{2}$ \\
\hline$: l=(011):(0 \pi 1)$ & $=* 98 \quad 58$ & $99 \quad 40-100 \quad 12$ & - \\
\hline$: m=(012):(110)$ & $=-$ & $735-7316$ & $73 \quad 12 \frac{1}{2}$ \\
\hline$l: m=(011):(110)$ & $=$ & $6411-6432$ & 6423 \\
\hline$m: \underline{m}=$ (Zwilling) & $=$ & $42^{\circ} 36^{\prime}$ & 4230 \\
\hline
\end{tabular}

Hieraus ergiebt sich das Axenverhältniss

$$
a: b: c=0,68407: 1: 1,1910 \text {. }
$$

Noch 15 Krystalle wurden gemessen, welche mit diesen ziemlich ubereinstimmende Werthe gaben, doch variirte der Winkel des Brachydomas etwas stärker. Diese Verschiedenheit des Domenwinkels, die an guten Krystallen sogar $\frac{1}{2}^{0}$ ubersteigen kann, muss durch Ungleichheiten in der Mineralsubstanz begründet sein. Bei dieser Gelegenheit ist zu bemerken, dass Breithaupt die Fundamentalwinkel des »Dalarnits « zu $68^{\circ} 59^{\prime}$ fur das Prisma und $100^{\circ} 44^{\prime}$ fur das Doma angiebt; da diese Werthe ganz ausserhalb der von mir beobachteten Werthe liegen, kommt vielleicht noch ein vierter Typus vor.

Da die ersten vor mehreren Jahren ausgefuhrten Analysen etwas schwankende Werthe gaben, lag die Annahme nabe, dass dies in Verunreinigungen seinen Grund hätte, was schon dadurch wabrscheinlich wird, das die Krystalle in Magnetkies liegen und oftmals einen dunnen Ueberzug von diesem Minerale tragen. Da dieser Arsenkies immer etwas $\mathrm{Ni}$ und $\mathrm{Co}_{\text {. }}$ enthält, schien es sogar plausibel, dass diese Metalle aus dem Muttergesteine stammen, was jedoch die Analyse nicht bestätigte. Der Magnetkies ist nämlich ganz frei davon, enthält aber Spuren von Mangan. Zur näheren Untersuchung dieser Frage habe ich unter verschiedenen Bedingungen Aetzversuche gemacht. Wie bekannt, ist Magnetkies in concentrirter Salzsäure löslich, während Arsenkies nicht angegriffen werden soll. Eine Anzahl Krystalle, die zufälligerweise fúnf Jahre lang in einer geschlossenen Glasflasche in concentrirter Salzsäure gelegen, war an den Krystallflächen nicht merkbar angegriffen.

Alle Krystalle dieses Typus geben nun sogleich beim Behandeln mit Salzsäure eine eisenhaltige Lösung, und zuweilen beobachtet man eine Entwickelung von Schwefelwasserstoff. Die in Lösung gegangene Eisenmenge ist jedoch sehr gering. Ein 1,4 g schwerer Krystall lieferte bei derartiger Behandlung mit warmer Salzsäure während einiger Minuten 0,1\% $F e$ in der Lösung, bei nochmaligem Behandeln wurde keine wägbare Menge gelöst. In derselben Weise verhalten sich die meisten Krystalle. An einem Paar besonders unebener Krystalle konnte ich recht viel Magnetkies heraus- 
lösen, welcher theils in Körnern, theils in Adern vorhanden war. Das mehrmals beobachtele Faclum, dass die Krystallflächen nach derartiger Behandlung heller und besser spiegelnd werden, erklärt sich dadurch, dass der Magnetkiesüberzug in diesem Falle gelöst wird.

Gepulverter Arsenkies lieferte ebenfalls eine eisenhaltige Lösung, die jedoch nie Arsenik enthält, noch wird Schwefelarsen gefällt. Der früher angefuhrte Krystall wurde grob gepulvert; nach 24 Stunden waren 0,12\% $\mathrm{Fe}$ von kalter conc. Salzsäure herausgelöst. Als $10 \mathrm{~g}$ Krystalle hierauf während 5 Minuten mit conc. Salzsäure behandelt wurden, bekam ich $0,05 \mathrm{~g}$ Eisenoxyd aus der Lösung, aber keine Spur von Nickel oder Kobalt. Hieraus ergiebt sich, dass der $\mathrm{Ni}$ - und $\mathrm{C}_{0}$-haltige Arsenkies nicht angegriffen wird.

Da aus diesen Versuchen als böchst wahrscheinlich hervorging, dass Magnetkies sogar in den Krystallen vorkommen konnte, wurden mehrere Krystalle mit Salpetersäure geätzt, von welcher sie je nach der Concentration schnell oder langsam gelöst wurden. Wenn eine 40 procentige Säure benutzt wird, so entwickeln sich die nitrösen Gase so langsam, dass man deutlich sieht, wie die Lösung nach ganz wenigen Linien rings um den Krystall vorwärts schreitet. Oftmals sind diese beinahe dem nicht auftretenden Brachypinakoid $\{010\}$ parallel. Versuche, die angestellt wurden, um zu finden, ob das in dieser Weise Gelöste eine von dem Restirenden abweichende Zusammensetzung besass, ergaben keine sicheren Anhaltspunkte.

Untersucht man dergleichen Krystalle genauer, so zeigt es sich, dass die Säure drei bis vier Furchen auslöste, welche oftmals rings um die vier Flächen verfolgt werden können, die an der längeren Axe zusammentreffen. Da die Krystalle vorher von Fett und Staub gereinigt waren, kann dies nicht zufällig sein, sondern es muss durch Einmengung einer von Salpetersäure leichtlöslichen Substanz, meist wohl Magnetkies, möglicherweise auch Nickelin (NiAs) begründet sein.

Die Aetzfiguren auf $\{011\}$ sind rund oder oval; in letzterem Falle immer mit ihrer längeren Axe parallel $\{001\}$. Am leichtesten wird das Doma $\{011\}$ angegriffen, das dabei eine wellenförmige, glänzende Fläche bekommt, mit nach der a-Axe parallelen Wellen; während das Prisma rauh wird, mit tiefen Gruben, die schief gegen diese Flächen, aber parallel $\{011\}$ liegen.

Alle Analysen wurden an vorher äusserlich mit $\mathrm{HCl}$ gereinigten Krystallen ausgefuhrt. I. A. eines grösseren, sehr spiegelnden Krystalles, der in Salpetersäure gelöst wurde, II. A. mehrerer kleinerer Krystalle, wovon ein Theil a. mit Alkalicarbonat geschmolzen, der andere Theil b. in Salpetersäure gelöst und der Schwefel nach Lun ge bestimmt wurde, III. A. eines Krystalles, der zuerst gepulvert und, nachdem das Pulver mit $\mathrm{HCl}$ gereinigt war, mit $\mathrm{HNO}_{3}$ gelöst wurde. 


$\begin{array}{lcccc} & \text { I. } & \text { II a. } & \text { II b. } & \text { III. } \\ \mathrm{Fe}\left(\mathrm{CoN}_{\mathrm{N}}\right) & 34,86 & (34,44) & - & 34,26 \text { (wovon } 0,57 \mathrm{Co}, 0,45 \mathrm{Ni} \text { ) } \\ \mathrm{S} & - & 19,96 & 19,72 & - \\ \mathrm{As} & - & 45,96 & - & 46,02\end{array}$

Die kleinen Krystalle (II.) und das gereinigte Kryslallpulver (III.) hatten eine Zusammensetzung, welche der Formel $(\mathrm{Fe}, \mathrm{Co}, \mathrm{Ni})(\mathrm{S}, \mathrm{As})_{2}$ entspricht; das Atomverhältniss $(\mathrm{Fe}, \mathrm{Co}, \mathrm{Ni}): S: A s$ ist nämlich in diesen Analysen 1: 1,005: 0,994. In der ersten Analyse ist dagegen der Eisengehalt ein wenig höher, was von einem beigemengten eisenreichen Minerale (Magnetkies) herrührt.

\section{Wester-Silfberg, Typus II.}

In derselben Grube, wo die soeben beschriebenen Krystalle vorkommen, findet man auch zwei andere Varietäten von Arsenkies. Von diesen fand ich den vorliegenden Typus, der Nr. I sehr ähnelt, in einem Gemenge von Kalkspath und Flussspath, und den anderen Typus (Nr. III), der sehr abweichend ist, zusammen mit Kalkspath und Zinkblende.

Bei den als Typus II bezeichneten Krystallen treten die Flächen $m\{110\}$, $l\{011\}$ und selten auch $s\{012\}$ auf. Die Ausbildung ist dieselbe, wie bei dem vorhergehenden Typus, doch sind Krystalle kurzprismatisch nach der $a$-Axe die allergewöhnlichsten (Taf. I, Fig. 3). Zwillinge wurden nicht beobachtet, dagegen kommen Nester von unregelmässig zusammengehäuften Individuen zuweilen vor. Der Glanz ist stark und die Farbe heller als bei vorigem Typus. Die Krystalle, die sehr klein, höchstens ein paar Millimeter lang sind, treten immer idiomorph ausgebildet auf, und liegen in Manganocalcit oder in weissem und grauem Flussspath. Ausser Arsenkies enthält dieses Gemenge auch $M n$-reichen Magnetit und Bleiglanz. Der Arsenkies ist das einzig krystallisirte Mineral; er ist älter als der Calcit und Flussspath, aber gleichzeitig mit dem Bleiglanz gebildet. Dieser Typus ist sehr selten.

Das specifische Gewicht ist bei $+18^{0}=6,07$. Die Messungen wurden an sieben Krystallen bewerkstelligt.

$\begin{array}{llll}\text { Flächen: } & \text { Fund.-Winkel: } & \text { Grenzwerth: } & \text { Ber. Winkel : } \\ m: m=(110):(1 \bar{T} 0)={ }^{*} 68^{0} 40^{\prime} & 68^{0} 28^{\prime}-68^{0} 47^{\prime} & - \\ l: l=(011):(0 T 1)={ }^{*} 100 \quad 1 \frac{1}{2} & 9950-1007 & - \\ l: s=(011):(012)= & - & 198-1923 & 19013^{\prime} \\ l: m=(011):(110)= & - & 6424-6428 & 6424\end{array}$

Hieraus wird das Axenhältniss $a: b: c=0,68300: 1: 1,19298$ berechnet.

Mit diesen Krystallen wurden zwei Analysen ausgefuhrt und zwar nach beiden früher beschriebenen Methoden. Die Krystalle zu der Analyse II waren vorher mit conc. Salzsäure behandelt, welche eine Lösung gab, die 
einen nicht bestimmbaren Gehalt von Eisen enthielt. Diese Krystalle geben keine Reaction auf Nickel und Kobalt und unterscheiden sich dadurch vom Typus I.

$\begin{array}{lcc} & \text { Analyse I. } & \text { II. } \\ F e & (34,94) & 34, \mathbf{2 6} \\ S & 19,86 & - \\ A s & - & 46,00\end{array}$

Aus diesen Analysen wild das Verhältoiss $F e: S: A s=1: 1,015:$ 1,001 berechnet, daher diese Krystalle genau die einfacbste Formel $\mathrm{Fe} S \mathrm{As}$ repräsentiren.

\section{Wester-Silf berg, Typus III.}

Dieser Arsenkies ist nur zusammen mit Magnetit und Blende gefunden worden. In der früher beschriebenen Grube tritt auch eine mächtige Einlagerung, hauptsächlich Flussspath und Kalkspath entbaltend, auf. Hier und da kommt auch Zinkblende, Magnetit und ziemlich heller Augit vor. Von Blende habe ich einige sehr kleine Krystalle angetroffen, die von den beiden Tetraëdern begrenzt sind, oftmals aber bildet dieses Mineral grossblätterige, gerundete Körner. Recht häufig sind Augitkrystalle; von einer derben Unterlage ragen kleine Prismen, $\{110\},\{100\}$ und $\{010\}$, in Kalkspath heraus. Der Magnetit ist körnig; in mikroskopischen Präparaten habe ich jedoch Krystalle gesehen.

Der Arsenkies krystallisirt in silberweissen Nadeln, nach dem Prisma verlängert. Die Krystalle sind besonders spröde, und die Winkel der Prismenzone variiren viel stärker, als von den kleinen, stark spiegelnden Flächen zu erwarten ist. Diese Eigenthtimlichkeiten rühren von Magnetiteinschlussen ber.

Einige Dunnschliffe des Gesteines, welches den Arsenkies beherbergt, wurden angefertigt, und diese Schliffe waren für die ganze Auffassung des vorliegenden Minerals entscheidend. Unter dem Mikroskope ist es eine sebr leichte Aufgabe, die genannten Mineralien zu unterscheiden, denn der Arsenkies reflectirt das Licht mit eisengrauer, der Magnetit mit blauschwarzer Farbe. Man sieht, wie vollkommen idiomorphe Krystalle von jenem Minerale sowohl in Magnetit, wie zuweilen auch in Zinkblende eindringen. Gleichfalls wird der Arsenkies ganz und gar von Magnetitpartikeln jeder Grösse durchsetzt; sehr sparsam sind dagegen Quarzeinschlüsse. Kein einziger Krystall ist von solchen fremden Mineralien ganz frei. An einem sehr grossen Arsenkieskrystalle sind die beiden Enden von einer Schaar Magnetitkörner gleichsam aufgespalten, und rings herum liegen Fragmente von dem Krystalle, was natürlich auf einer ganz gleichzeitigen Entstehung der beiden Mineralien beruht.

Von diesem Typus wurden nur die Formen $m\{110\}, l\{011\}$ und $s\{012\}$ 
beobachtet (Fig. 4). Penetrationszwillinge nach $m\{110\}$ sind nicht ungewöhnlich.

Die Nadeln sind $1-2 \mathrm{~cm}$ lang, ihre Breite überstieg selten $1 \mathrm{~mm}$. Sehr oft sind mehrere Krystalle in paralleler Stellung nach $\{010\}$ verwachsen; in dieser Weise entstehen breite, längs des Prismas tief gefurchte Blätter. In der Regel sind die mittleren Individuen am dicksten, die äusseren immer schmäler. Da sie ganz eingewachsen sind, gaben nur die äussersten Reflexe, die zur Messung brauchbar sind, während die Mitle eines solchen Krystallstockes abgerundet ist und auch die Krystallenden dadurch axtförmig gekrümmt werden. Doch kommen auch isolirte Individuen vor.

Zehn der besseren Krystalle, wovon jedoch nur drei in der Brachyzone messbar waren, gaben die folgenden Werthe.

$\begin{array}{llll}\text { Flächen: } & \text { Fund.-Winkel: } & \text { Grenzwerthe: } & \text { Ber. Winkel : } \\ m: m=(110):(1 \mathrm{~T} 0)={ }^{*} 67050^{\prime} & 67^{0} 37^{\prime}-68^{\circ} 15^{\prime} & - \\ l: l=(011):(0 T 1)={ }^{*} 9954 & 9945-9955 & - \\ l: s=(011):(012)=- & 192-1918 & 19015^{\prime} \\ l: m=(011):(110)=- & 659 & & 6443\end{array}$
rechnet.

Hieraus wird das Axenverhältniss $a: b: c=0,67239: 1: 1,1896$ be-

Das spec. Gew. wurde bei $0^{0} \mathrm{zu} 6,11$ gefunden, doch wurde hierbei nur 0,285 g Substanz benutzt.

Alle Analysen zeigen Spuren von Mangan, welches von den Magnetiteinschlüssen stammt; wie ich früher gezeigt, ist nämlich der Magnetit stark manganhaltig*). Von gröberen Magnetitkörnern wurde das pulverisirte Mineral in I. und II. mit einem Magnet gereinigt und alsdann mit Salzsäure und Kaliumchlorat zersetzt; in III. wurde das Krystallpulver ohne vorheriges Behandeln mit Alkalicarbonat und Nitrat aufgeschlossen.

\begin{tabular}{lrrc} 
& \multicolumn{1}{c}{ I. } & \multicolumn{1}{c}{ II. } & III. \\
$F e$ & 34,72 & 34,60 & $(35,00)$ \\
$M n$ & 0,18 & 0,20 & - \\
$S$ & 18,22 & 17,93 & 18,53 \\
$A s$ & $-46,60$ & 46,32 & -
\end{tabular}

Das Verhältniss $F e: S: A s$ ist hiernach im Mittel $=1: 0,919: 0,996$.

Hier liegt also entschieden eine Ausnahme von der Formel $\mathrm{Fe}(\mathrm{S}, \mathrm{As})_{2}$ vor, ganz ubereinstimmend mit dem, was Arzruni für mehrere Arsenkiese angiebt. Doch hat die mikroskopische Untersuchung gezeigt, dass dies von den kleinen Magnetiteinschlüssen herrührt, und eine Berechnung zeigt, dass

*) Tschermak's min.-petrogr. Mitth. 1885, S. 110. 
eine Einmengung von 1,7\% Magnetit, entsprechend 1, $2 \% \mathrm{Fe}$, die hier beobachtele Abweichung exklärt. Gewiss werden die grösseren Körner dieses Minerals von einem Magnet ausgezogen, aber kleinere bleiben immer noch zurúck, was úbrigens theils durch den $M n$-Gehalt, theils durch die Verluste in den Analysen sich zeigt, welche daher kommen, dass ein Theil des in den Analysen als Eisen Berechneten in der That als Oxydoxydul (Magnetit) vorhanden ist. Ob eine Analyse an sorgfältig sowohl mit dem Magneten, als mit Salzsáure gereinigtem Pulver sichere Resultate geben wúrde, ist fraglich, denn theils kommt der Magnetit in sehr kleinen Körnern vor, theils scheint dieser Arsenkies angegriffen zu werden, wenn er eine Zeit lang mit Salzsäure digerirt wird.

\section{Spräkla-Kalkbruch, Typus I.}

Etwa 2 km östlich von dem Bergrucken, in welchem die Gruben WesterSilfbergs bauen, liegt das ziemlich enge Spräkla-Starbo-Thal, wo ein Paar bedeutende Einlagerungen von körnigem Kalk in dem herrschenden Granulite vorkommen. Diese Felsart, sowie der Kalkstein haben ihr Streichen von N. - S. und beide fallen steil gegen $O$. Nur in einem sủdlichen, bei Spräkla liegenden Bruche habe ich krystallisirten Arsenkies gefunden.

Der Kalkstein ist recht grobkörnig und in der Regel ziemlich rein; sehr gewöhnlich kommt als accessorischer Bestandtheil schwarze Hornblende vor; dieses Mineral bildet lange, band- oder linienförmige Einlagerungen in dem Gesteine, und hier tritt dichter Arsenkies auf. Uebrigens habe ich folgende Mineralien beobachtet: Granat, Zinkblende, Magnetkies, Kupferkies, Bleiglanz, sowie Krystalle von Augit und Calcit. Die Calcitkrystalle kommen nur in später gebildeten Spalten vor, sind meist geätzt und zeigen gewöhnlich die Formen $R$ und $R 3$.

Arsenkieskrystalle Typus I kommen mit dem Augit vor, der gewöhnlich dicht ist; wenn eine grössere Menge von diesem Minerale auftritt, besteht der innere Kern gewöhnlich aus Hornblende. Die Augitkrystalle haben nur die Prismenzone deutlich ausgebildet, so dass nur $\{110\}\{010\}\{100\}$, eine Querfläche und zwei Pyramidenflächen zu sehen sind. Eine Analyse von diesem lauchgrünen Augit wurde von Hilda Weibull ausgefuhrt und gab :

\begin{tabular}{lr}
$\mathrm{SiO}_{2}$ & 49,61 \\
$\mathrm{CaO}$ & 23,20 \\
$\mathrm{FeO}$ & 11,71 \\
$\mathrm{MgO}$ & 10,19 \\
$\mathrm{Al}_{2} \mathrm{O}_{3}$ & 4,84 \\
$\mathrm{Fe}_{2} \mathrm{O}_{3}$ & 0,42 \\
\hline & 99,97
\end{tabular}




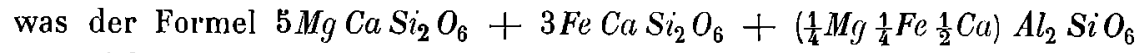
entspricht.

Der Arsenkies ist oft wohlausgebildet, von wechselnder Grösse und glänzend silberweiss; - er ist älter als der Calcit, aber gleichzeitiger Entstehung mit dem Augit. Die meisten Arsenkieskrystalle sitzen nämlich an und in diesem Minerale; zuweilen findet man jedoch kleine Augitkörner in ganz idiomorphem Arsenkies.

Die beobachteten Formen sind: $m\{110\}, l\{011\}, s\{012\}, u\{023\}$ und $c\{001\}$. Die beiden letztgenannten Formen, von denen $u$ für Arsenkies neu ist, traten jedoch nur je an einem Krystalle auf. Der Habitus variirt bedeutend, doch herrschen Krystalle kurzprismatisch nach $m\{110\}$ (Fig. 5) vor. Contactzwillinge nach dieser Fläche sind nicht ungewöhnlich.

$$
\begin{aligned}
& \text { Flächen: Fund.-Winkel: Grenzwerthe: Ber. Winkel: } \\
& \begin{array}{lccc}
m: m=(110):(1 T 0)={ }^{*} 68^{0} 29 \frac{1}{2}^{\prime} & 69^{0} 25^{\prime}-68^{0} 40^{\prime} & - \\
l: l=(011):(011)={ }^{*} 9957 & 9945-9959 & - \\
l: s=(011):(012)= & - & 19^{\circ} \cdot 8^{\prime} & 190121^{\prime} \\
l: u=(011):(023)=- & 1140 & 1132 \\
m: l=(110):(011)=- & 64032^{\prime}-64037^{\prime} & 6428 \frac{1}{2} \\
m: m^{\prime}=(\text { Zwillinge })=- & 43^{\circ} 45^{\prime} & 431
\end{array}
\end{aligned}
$$

Hieraus wird das Axenverhältniss $a: b: c=0,68077: 1: 1,19070$ berechnet.

Die meisten Krystalle sind ganz homogen, doch bleibt oftmals beim Behandeln mit Salpetersäure ein ungelöster Rückstand von Augit. In der Analyse I. wurde mit Alkalicarbonat zersetzt; in II. wurden die Krystalle vor der Analyse gepulvert, mit $\mathrm{HCl}$ behandelt, wobei eine nicht wägbare $\mathrm{Fe}$-Menge in Lösung gegangen war; nachber wurde mit $\mathrm{HNO}_{3}$ gelöst, wobei eine geringe Menge Augit zuruckblieb.

Analyse I.

$\begin{array}{lcc}F e & (34,75) & 34,44 \\ S & 19,37 & - \\ A s & - & 45,38 \\ \text { Ungelöstes } & - & 0,18\end{array}$

II.

, 38

0,18

Das Verhältniss $F e: S: A s$ ist danach $1: 0,985: 0,984$ und die Formel also $\mathrm{FeSAs}$.

\section{Spräkla-Kalkbruch, Typus II.}

Noch ein anderer Typus von Krystallen kommt bei Spräkla vor. Eine goniometrische Untersuchung der bei verschiedener Gelegenheit gefundenen Krystalle zeigte nämlich, dass die im reinen Calcit sitzenden Krystalle etwas andere Constanten besitzen, als die zusammen mit Hornblende oder Augit vorkommenden. 
Diese Krystalle zeigen folgende Formen: $m\{110\}, e\{101\}, l\{011\}$, $s\{012\}$ und $k\{021\}$. Im allgemeinen Habitus ähneln sie dem vorhergehenden Typus, doch sind sie durchschnittlich elwas mehr nach der c-Axe verlängert (Fig. 6 und 7). Das spec. Gew. wurde zu 6,204 bei $25^{0}$ gefunden.

Die Messungen, die an sieben Krystallen bewerkstelligt wurden, stimmten sehr wohl überein und ergaben :

Flächen: Fund. -Winkel: Grenzwerthe: Ber. Winkel :

\begin{tabular}{|c|c|c|}
\hline$m: m=(110):(1 \bar{\top} 0)={ }^{*} 67041^{\prime}$ & $67034^{\prime}-67046^{\prime}$ & - \\
\hline$l: l=(011):(0 T 1)=-$ & $100^{0} 3^{\prime}$ & $99^{\circ} 45^{\prime}$ \\
\hline$: l=(012):(011)=$ & 199 & $1911 \frac{3}{4}$ \\
\hline$: l=(021):(011)=$ & 1623 appr & $1714 \frac{1}{2}$ \\
\hline$: e=(101):(\bar{T} 01)=121$ & $120^{\circ} 59^{\prime}-1210^{\prime} 7^{\prime}$ & - \\
\hline$n: e=1$ & $4331-4335$ & $43 \quad 41 \frac{1}{2}$ \\
\hline$m: l=(110):(011)=$ & $6428-6456$ & $64 \quad 48$ \\
\hline$m: s=(110):(012)=$ & $7320-7326$ & $7329 \frac{3}{1}$ \\
\hline
\end{tabular}

Hieraus wird das Axenverhälıniss $a: b: c=0,6705: 1: 1,1863$ berechnet.

Für die chemische Untersuchung hatle ich nur etwa $\frac{1}{3} \mathrm{~g}$ Krystalle aus einem älteren Funde ubrig; die im letzten Jahre gefundenen gehören nämlich alle zum Typus I. Diese Krystalle, die vorher gemessen wurden, enthalten jedoch etwas Magnetit, welcher dadurch zum grössten Theile entfernt wurde, dass ich das Pulver mit $\mathrm{HCl}$ digerirte. Zur Analyse verfugte ich nur uber $0,2726 \mathrm{~g}$, die mit Alkalicarbonat und Nitrat zersetzt wurden, daher nur der Eisen- und Schwefelgehalt festgestellt werden konnte:

$\begin{array}{ll}\text { Fe } & 34,11 \\ S & 17,49\end{array}$

Berechnet man den Verlust als $A s$, so ergiebt sich das Verhăltniss $F e$ : $S: A s=1: 0,901: 1,068$. Da hier nur eine Analyse vorliegt, ist zu bemerken, dass aller Wahrscheinlichkeit nach der $\mathrm{S}$-Gehalt richtig, der $\mathrm{Fe}$ Gehalt etwas zu hoch gefunden ist - wegen der Methode und eventueller Einschlüsse - und dass dadurch für den Arsengehalt eine entsprechend zu niedrige Zahl erhalten wurde.

\section{Nybergs-Gruben.}

Etwa $4 \mathrm{~km} 0$. von Wester-Silfberg, gegenüber dem soeben beschriebenen Starbo-Thale, liegen an einem zweiten Bergrucken die NybergsGruben, die auf Magnetit bauen. In der granulitischen Felsart liegen hier und da Einlagerungen von Augitfels und in diesem Gesteine kommt das Erz vor. Der Augit ist oft in Chlorit und Serpentin umgewandelt; ausser diesen Mineralien habe ich Asbest, Galcit, Granat, Epidot, Hornblende und Pyrit beobachtet. Nur von den drei letzteren liegen Krystalle vor. 
Als dichte Körner findet man zuweilen den Arsenkies im Erze, von Krystallen wurden nur wenige an diesem Fundorte angetroffen. Der Arsenkies liegt mit dem Pyrit in einem Gemenge von Magnetit und etwas zersetzlem Augit. Die Krystalle sind ganz klein, und jedes Individuum ist ringsum ausgebildet, zeigt aber keine anderen Formen als $m\{110\}$ und $l\{011\}$, von welchen gewöhnlich letztere vorherrscht. Die Ecken sind öfters gerundet und die Flächen etwas rauh. Vier gute Krystalle, die gemessen wurden, gaben folgende Winkel:

$\begin{array}{lllc}\text { Flachen: } & \text { Fund.-Winkel: } & \text { Grenzwerthe: } & \text { Ber. Winkel : } \\ m: m=(110):(1 T 0)={ }^{*} 68^{0} 22^{\prime} & 68^{0} 20^{\prime}-68^{0} 37^{\prime} & - \\ l: l=(011):(0 T 1)={ }^{*} 9956 & 9952-1001 & - \\ m: l=(110):(011)=\quad- & 6421-6438 & 6403 l^{\prime}\end{array}$

Das Axenverhältniss ist also : $a: b: c=0,6792: 1: 1,1910$.

Wenn man diese Krystalle mit Salzsäure behandelt, geben, sie sogleich eine gelbe Lösung, ein Zeichen, dass Magnetit zugegen ist. Fur die Analyse I. und II. wurden etwa 0,8 g Krystalle grob pulverisirt. Das Pulver wurde einige Stunden lang kalt mit verdunnter Salzsäure digerirt, wobei $0,66 \%$ $F e$ ausgezogen wurde. Der grösste Theil desselben stammt sicherlich von eingesprengtem Magnetit. Von diesem Mineralpulver wurde ein Theil mit Salpetersïure (I.), der andere mil Alkalicarbonat (II.) aufgeschlossen. In Analyse III. wurde mit dem Magnet gereinigtes Pulver mit Alkalicarbonat und Nitrat aufgeschlossen.

$\begin{array}{lccc} & \text { I. } & \text { II. } & \text { III. } \\ F e & 34,23 & - & 34,68 \\ S & - & 19,00 & 18,99 \\ \text { As } & 4.6,76 & - & - \\ \text { Ungelöstes } & 0,22 & - & -\end{array}$

Aus diesen Analysen ergiebt sich das Verhältniss $F e: S: A s=1$ : $0,987: 1,018$.

\section{Sala-Grube.}

Dieser Fundort ist, wie ich früher nachgewiesen habe, von einem ganz besonderen Interesse. Die davon erhaltenen Krystalle zeigen nämlich nach Arzruni und Bärwald eine Zusammenselzung, welche am meisten von der jetzt gewöhnlich angenommenen Formel $\mathrm{Fe}(\mathrm{S}, \mathrm{As})_{2}$ abweicht. Ich habe mich daher bestrebt, diese Krystalle eingehender zu untersuchen, und glutcklicherweise bekam ich von dem Grubendirector Heberlé ein genügendes Material von dem in Sala gewöhnlichen Typus von Arsenkies.

Diese Krystalle sitzen alle in zerselztem Augit, sogenanntem Pikrophyll; die von den genannten Forschern untersuchten Exemplare waren theils in Serpentin (Pikrophyll ?), theils im körnigen Kalke gefunden. Eine 
Differen $z$ im Habilus und Winkel zwischen denselben wurde jedoch nicht beobachtet.

Uebereinstimmend mit Arzruni und Bärwald fand ich nur die Flächen von $m\{110\}, l\{011\}$ und $s\{012\}$. Die Krystalle zeigen Mittelgrösse und waren von prismatischem Habilus nach $m\{110\}$. Zwillinge sind sehr bäufig sowohl nach $m\{110\}$, als nach dem nicht vorkommenden $\{101\}$. Sogar Drillingskrystalle nach dieser Fläche sind häufig. Die einfachen Individuen ahneln den früher unter Wester-Silfberg III (Fig. 4) beschriebenen.

Die Krystalle sind rein weissglänzend, die Flächen jedoch oft nicht ganz eben; auch wenn sie ganz tadellos erscheinen, stimmt die Messung an einem Krystalle nicht gut mit der an einem anderen uberein. An acht der besten Krystalle habe ich folgende Winkel gemessen.

Flächen: Fund.-Winkel: Grenzwerthe: Ber. Winkel :

\begin{tabular}{|c|c|c|c|c|c|c|}
\hline$m$ & $: m=$ & $(110):(1 \pi 0)$ & $=$ & ${ }^{*} 68^{0} 44^{\prime}$ & $68^{\circ} 23^{\prime}-69^{0} 9^{\prime}$ & 一 \\
\hline$l$ & $: l=$ & $(011):(0 T 1)$ & $=*$ & $* 10027$ & $10026-10028$ & \\
\hline$l$ & $: s=$ & $(011):(012)$ & $=$ & - & $196-1916$ & $19014^{\prime}$ \\
\hline$s$ & $: s=$ & $(012):(012)$ & $=$ & 一 & $620 \quad 0^{\prime}$ & 6159 \\
\hline$l$ & $: l=1$ & Iwill. n. $(101)$ & $=$ & - & 3720 & $36 \quad 54 \cdot \frac{1}{2}$ \\
\hline & $=$ & $-\quad-(104)$ & $=$ & 一 & 5023 & $50 \quad 11 \frac{1}{2}$ \\
\hline & $: \underline{m}=$ & $-(110)$ & $=$ & - & 4252 & 4232 \\
\hline
\end{tabular}

Hieraus wird das folgende Axenverhältniss $a: b: c=0,68386: 1$ : 1,2013 berechnet.

Arzruni und Bärwald fanden folgende Winkel :

$$
\begin{aligned}
& m: m=(110):(1 T 0)=68^{0} 29^{\prime} \\
& l: l=(011):\left(0 T^{\top} 1\right)=9955 \frac{1}{2} \\
& l: s=(011):(012)=197 \\
& s: s=(012):\left(0 T^{\top} 2\right)=6140 \frac{1}{2}
\end{aligned}
$$

woraus sie das Axenverhälıniss $a: b: c=0,68066: 1: 1,19017$ berechneten.

Zur Analyse wurden 1,3 g Krystalle gepulvert und darnach mit $\mathrm{HCl}$ ein Paar Stunden behandelt. Dabei wurde die Lösung sogleich etwas gelblich, daher also eine Ferriverbindung gelöst wurde, die im Ganzen 0,2 \% $F e$ enthielt. Das gewaschene und getrocknete Pulver wurde in fruher beschriebener Weise analysirt. Beim Behandeln mit $\mathrm{HNO}_{3}$ (I.) blieb ein Ruckstand von 2,01\% zurück, welcher sich als Pikrophyll erwies.

I.

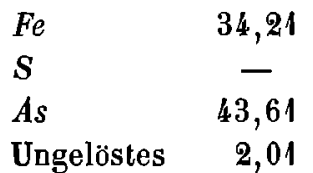

Ungelöstes $\quad 2,01$
II.

$(34,78)$

19,98

Rechnet man den ungelösten Ruckstand ab, so hatte das Mineral folgende Zusammensetzung : 


$$
\begin{array}{ll}
\text { Fe } & 34,92 \\
S & 20,39 \\
\text { As } & 44, \mathbf{5 1} \\
\cline { 2 - 2 } & \mathbf{9 9 , 8 2}
\end{array}
$$

woraus das Verhältniss $F e: S: A s=1: 1,022: 0,952$ folgt.

Später habe ich auch ein Exemplar aus einer älteren mineralogischen Sammlung an der Universität zu Lund gefunden, wo der Arsenkies in einer aus Kalk und Talk bestehenden Stufe sass. Die meisten Krystalle waren nicht zur Messung geeignet, denn theils waren die Flăchen matt, theils zeigten sie einen schaligen Bau nach dem Prisma. Ein Paar von den grösseren, nicht vollkommen ausgebildeten Krystallen hatten einen feinkörnigen Bruch, der theilweise von braungelber Farbe war (von Magnetkies ?). Zwillinge nach $\{101\}$ sehr gewöhnlich.

Nur sechs Krystalle waren zur Messung brauchbar; die drei besseren von diesen gaben folgende Werthe, welche jedoch nicht als gut bezeichnet werden können :

$$
\begin{aligned}
& m: m=(110):(1 \mathrm{~T} 0)=68^{0} 36^{\prime}-68^{0} 56^{\prime} \\
& l: l=(011):(0 \overline{1})=100 \quad 0-10028 \\
& l: s=(011):(012)=192-1920
\end{aligned}
$$

was ziemlich wohl mit früheren Messungen an diesem Minerale stimmt.

Die Analyse dieser Krystalle ergab:

$$
\begin{array}{lr}
F e & 34,55 \\
S & 19,85 \\
A s & 45,03 \\
\text { Ungelöstes } & 0,35 \\
\cline { 2 - 2 } & 99,79
\end{array}
$$

Hieraus berechnet sich das Verhältniss $F e: S: A s=1: 1,004: 0,973$.

Aus dieser Analyse gebt hervor, dass der bei Sala gefundene Arsenkies eine allerdings etwas schwankende Constitution hat, dass aber die Formel $\mathrm{Fe}(\mathrm{S}, \mathrm{As})_{2}$ an den von Inir unlersuchten Varietäten zutreffend ist.

\section{Vena-Grube, Typus I.}

Von dieser Grube (in Nerike) erhielt ich aus dem Reichsmuseum durch Freiherrn Prof. A. E. Nordenskiöld einige $1-3 \mathrm{~cm}$ grosse Krystalle. Ihr Habitus wechselt etwas, doch sind die meisten prismatisch nach der $a$-Axe. Die Brachydomen sind stark entwickelt und das Prisma trill sehr zurück. Die letztgenannten Flächen begrenzen den Krystall an den Enden, aber an zwei Individuen kam die Pyramide $v\{212\}$ hinzu.

Beobachtet wurden folgende Formen: $m\{110\}, l\{011\}, s\{012\}, k\{021\}$, sowie die soeben angeführte Pyramide $v\{212\}$. Von den Domen herrscht 
$l\{011\} ; l<\{021\}$, das sehr schmal ist, wurde nur an einem Krystalle angetroffen (Taf. 1, Fig. 8 und 9). Ein Krystall war ein Zwilling nach $\{101\}$.

Die Krystalle spalten gut nach $\{110\}$. An denselben, resp. ihren Bruchflächen habe ich die folgenden Mineralien beobachtet: Kupferkies, Chlorit und Quarz. Bei den Messungen konnten drei Krystalle benutzt werden, die folgendes Resultat gahen :

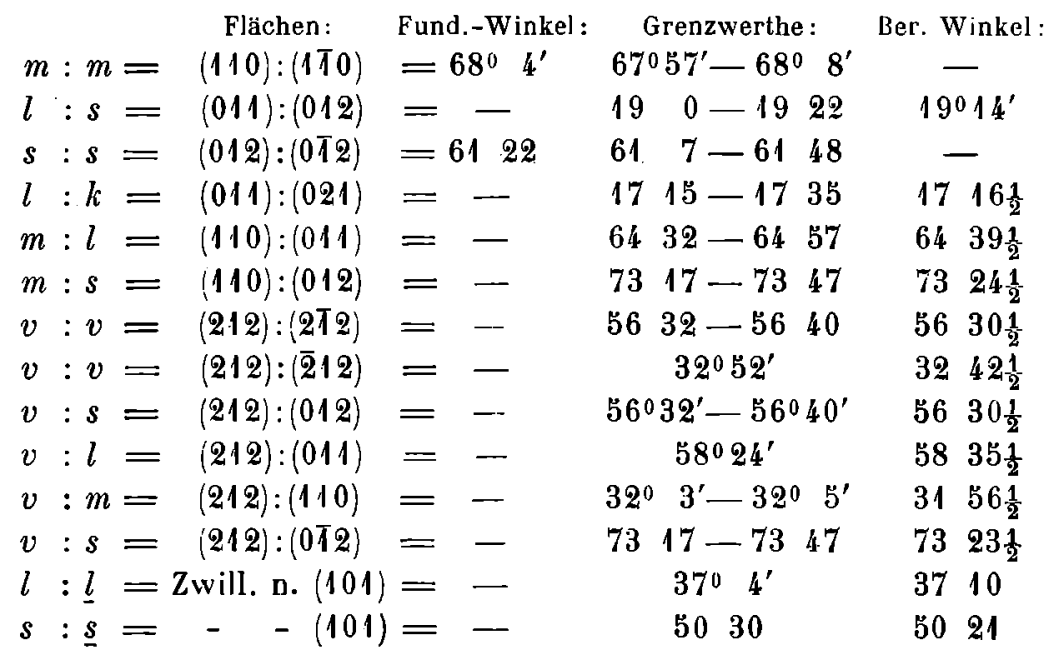

Aus den Fundamentalwinkeln ergiebt sich das Axenverhältniss $a: b: c=$ $0,67536: 1: 1,1867$.

Die chemische Untersuchung wurde zunächst nur mit Rücksicht auf den Schwefel- und Kobaltgehalt ausgeführt, denn ein exactes Resultat war nicht zu erwarten, da die für die Analyse geoplerten Krystalle nicht rein waren. Ausser Kupferkies und Ghlorit enthalten sie wahrscheinlich Magnetkies, denn das Pulver wurde unter $\mathrm{H}_{2} \mathrm{~S}$-Entwickelung von Salzsäure etwas angegriffen. Die Analysen sind in gewöhnlicher Weise ausgeführt und gaben :

I.

$\begin{array}{lcc}F e & 31,90 \\ C o & 2,50 & 34,32 \\ S & - & 18,16 \\ A s & 46,33 & - \\ \text { Nicht Gelöstes } & 0,82 & - \\ C u \text { unbedeutend, nicbt bestimmt. }\end{array}$

Das Verhältniss $(F e+C o): S: A s$ ist $=1: 0,926: 1,009$. Wegen der erwähnten Einschlusse ist der Metallgehalt und möglicherweise der Schwefelgehalt etwas zu hoch, während der Arsengehalt zu niedrig ist.

Zum Typus II kann ich einen fragmentarischen Krystall rechnen, dessen Prismenwinkel ganz abweichend gefunden wurde. Der fragliche Krystall 
ist ein Zwilling nach $m\{110\}$. Die beiden Individuen, die mil der Zwillingsfläche verwachsen sind, zeigen ausser den Prismen $\{110\}$ nur die Domenflächen $\{011\}$, welche parallel der $a$-Axe gefurcht sind.

Der Prismenwinkel $m: m=(110):(1 \bar{T} 0)$ war ubereinstimmend an beiden Individuen $69^{\circ} 30^{\prime}$, daraus berechnet sich der Zwillingswinkel $m: m^{\prime}$ zu $41^{\circ}$, während $41010^{\prime}$ gemessen wurde.

\section{Håkansboda-Grube.}

Den bekannten Glaukodot von Håkansboda habe ich bei dieser Gelegenheit ebenfalls untersucht. Obschon ich aus verschiedenen Sammlungen ansehnliches Material gehabt habe, kann ich doch nichts besonders Neues darubber liefern. Die ganz kleinen, gut messbaren Krystalle waren nämlich nie rein, und die grösseren mit ihren unebenen, gebrochenen Flächen sind nicht gut messbar. Wie bekannt, sitzt das Mineral in Kupferkies, daher trifft man auch uberall an den Krystallen Körner und Adern von diesem Minerale. Sogar kleine, äusserlich ganz feblerfreie Krystalle entbielten inwendig eine beträchtliche Menge davon.

Das Resultat meiner an zwei kleinen Individuen angestellten Messungen stimmt gut mit demjenigen von Lewis und Sadebeck*), denn ich fand das Prisma $m: m=(110):(1 \bar{T} 0)=69^{0} 14^{\prime}-69^{0} 21^{\prime}$ und das Doma $l: l=$ $(011):(0 T 1)=100^{\circ} 21^{\prime}$. Daher als sicher festgestellt anzusehen ist, dass der Glaukodot aus Håkansboda in der Regel ein Prisma von $69 \frac{1}{3} 0$ besitzt. Von den Krystallen, die ich benutzen konnte, war aus fruher angefuhrtem Grunde kein einziger zur Analyse verwendbar, weshalb ich von solcher Untersuchung Abstand genommen habe.

\section{Freiberg in Sachsen.}

Unter dem Namen "Freiberg, Kühschacht-Fundgrube " befindet sich im Mineraliencabinet zu Lund eine aus älterer Zeit herrührende Stufe. Das Mineral sitzt mit viel Pyrit in einer hellgrunen, serpentinisirten Gebirgsart. Die meisten Krystalle sind recht gross, prismatisch nach $m\{110\}$. Diese Fläche ist nicht ganz eben, sondern zeigt uberall kleine, mit dem umgebenden Minerale gefüllte Hohlräume. Die Prismen sind durch die Brachydomen $s\{012\}$ und $t\{013\}$ zugespitzt; diese sind jedoch nicht deutlich ausgebildet, sondern die beiden Enden sind rund und nach der $a$-Axe gestreift, so dass bei der Messung eine Reihe von Reflexen entsteht; die Winkeldistanz der äusseren derselben beträgt etwa $63^{\circ}$, die der inneren etwa 420 , was vollkommen den Winkeln (012):(012) und (013):(0T3) entspricht (Fig. 10).

Die allermeisten Krystalle sind Zwillinge nach $m\{110\}$. Viele haben

*) Tscherm. min.-petrogr. Mitth. 1877, S. 355. S. diese Zeitschr. 1, 67; 2, 518. 
deutlich pseudohexagonalen Habitus, was Fig. 11 in Projection auf die Basis zeigt. An zwei Krystallen, die gemessen wurden, war $m: m=(110):(1 \bar{\tau} 0)$ $=68^{\circ} 48^{\prime}-69^{\circ} 24^{\prime}$; der beste Werth war $69^{\circ} 8^{\prime}$. Der Winkel $m: m$ wurde an Zwillingskrystallen $41^{1} 26^{\prime}$ gefunden, berechnet $41^{0} 44^{\prime}$. Das Verhältniss $a: b$ wird also $0,6890: 1$.

Für die Analysen wurden 1,3 g pulverisirt und mit $\mathrm{HCl}$ behandelt; dabei wurden $0,35 \% \mathrm{Fe}_{2} \mathrm{O}_{3}+\mathrm{Al}_{2} \mathrm{O}_{3}, 0,2 \% \mathrm{CaO}$ und $0,05 \% \mathrm{MgO}$ gelöst. Der Rückstand wurde in gewöhnlicher Weise analysirt; in der Analyse I, wo mit $\mathrm{HNO}_{3}$ aufgeschlossen wurde, bekam ich 1,80\% Ungelöstes.

I.

$\begin{array}{lrc}\text { Fe } & 34,82 & - \\ S & - & 21,25 \\ \text { As } & 42,27 & - \\ \text { Ungelöstes } & 1,80 & -\end{array}$

Das Verhältniss $F e: S: A s=1: 1,069: 0,911$. Der geringe Ueberschuss von Eisen im Vergleiche mit den Metalloiden wird durch die Löslichkeit der Gebirgsart erklärt.

Von Freiberg hat Arzruni zwei verschiedene Arten von Arsenkies untersucht. Diese sind jedoch von den meinigen in Winkeln, Flächen und Gonstitution ganz verschieden. Da auch Frenzel*) Krystalle erwähnt, deren Flächen und $Z$ willingsbildung von allen diesen verschieden sind, so kennt man jetzt von diesem Fundorte wenigstens vier verschiedene Typen.

Die fruher erwähnten Forscher, zuerst Breithaupt, haben gezeigt, wie dieses Mineral von Fundort zu Fundort in Krystallform und Zusammensetzung wechselt. Meine Erfahrung in dieser Richtung geht noch weiter, denn kaum ein Mineral tritt an demselben Orte in so vielen Typen auf, wie dieses. Bei Wester-Silfberg habe ich in derselben Grube dre i Varietäten, bei Spräkla, wo das Mineral sehr sparsam vorkommt, z we i solche gefunden. Auch bei Sala, Freiberg, Håkansboda und Vena kommen mehr als eine Varietät vor.

Diese Typen sind in der Regel ganz constant, denn sie können oft nicht nur durch den Prismenwinkel und die Zusammensetzung, sondern auch durch die begleitenden Mineralien unterschieden werden. Doch sind die Typen nicht immer vollständig getrennt, sondern Uebergänge in gewisser Richtung sind nicht ausgeschlossen. Besonders ist hervorzuheben, dass die Individuen von demselben Typus bei Winkelmessungen gewisse Verschiedenheiten zeigen. Die Analysen dagegen, die in der Regel an mehreren Krystallen ausgefuhrt wurden, zeigen gewöhnlich diese Variationen nicht.

*) Sitzungsber. der Akad. d. Wiss. Wien 56, (I), 42. 
Die einzige ganz exacte Untersuchungsmethode wäre freilicb die, verschiedene Krystalle nach den Resultaten der Messung zu sortiren und nur die vollkommener übereinstimmenden zu analysiren. Diese Methode stelll jedoch allzu grosse Anforderungen an die Menge und Beschaffenheit des Materials, so dass sie gewöhnlich gar nicht durchführbar ist. Man muss oft die besten Krystalle messen und bei der Berechnung innerhalb der Grenzwerthe bei den Winkeln sich halten, die am meisten constant sind; nachher werden die in dieser Weise gemessenen Krystalle zur Analyse benutzt. Da wenig Material zu Gebote stand, mussten sogar schlecht messbare Krystalle von demselben Fundorte benutzt werden.

Es bleibt jetzt ubrig, die Anfangs erwähnte Frage näher zu erörtern : Welche Formel passt sich am besten den jetzt untersuchten Arsenkiesen an? Ist die Formel $\mathrm{Fe}(S, A s)_{2}$ - welche sowohl die einfachste ist, wie auch die nahe Verwandtschaft mit Löllingit und Markasit zeigt - die richtige oder muss die Constitution durch die complicirte Formel $\mathrm{Fe}_{2} \mathrm{~S}_{2}+x \mathrm{Fe}_{m} A s_{n}$ ausgedrückt werden, d. h. weicht das Verhältniss $F e:(S+A s)$ so viel von 1: $2 \mathrm{ab}$, dass dies nicht auf Analysen- oder anderen Fehlern beruht?

Wenn die vorliegenden Arsenkiese, wie es gewöhnlich geschieht, obne weiteres analysirt wurden, so geben einige von ibnen Werthe, die so bedeutend von dem Verhältnisse $F e:(S, A s)=1: 2$ abweichen, dass die erste Formel wenigstens fraglich ist, denn dieses Verhältniss ist z. B. bei WesterSilfberg III 1:1,915, bei Vena 1:1,935 und bei Nyberg 1:1,954. Doch ist im Vorhergehenden bewiesen, dass gerade diese Varietäten nicht unbeträchtliche Einschlússe enthalten, die alle den Eisengehall vermehren und die Summe der Metalloide herabsetzen. Zu diesen Varietäten gebört auch das Mineral von Håkansboda, das gerade deswegen nicht analysirt worden ist.

So weit ich gefunden habe, ist kein einziger von den hier beschriebenen Krystallen vollkommen frei von Einschlüssen. Am reinsten ist gewiss der Arsenkies von Wester-Silfberg Typus II, in welchem höchstens Spuren von Magnetit vorkommen. In allen anderen habe ich in irgend einer Form Einschlüsse nachgewiesen, obschon sie zuweilen, wenn man vorher mit Salzsäure behandelt oder wenn man das Mineral in Salpetersäure löst, zum Theil entfernt werden können.

Hieraus geht hervor, dass man nicht erwarten kann, dass alle Analysen die Conslitution der reinen Mineralsubstanz zeigen können. Das relative Verhältniss der Bestandtheile bei den reinen odergereinigten Mineralien ist in folgender Tabelle zusammengestellt.

$\begin{array}{lcccc} & \mathrm{Fe}(\mathrm{Co}): & S & A s & (S+A s) \\ \text { Wester-Silfberg II } & 1 & 1,015 & 1,001 & 2,016 \\ \text { Nyberg } & 1 & 0,987 & \mathbf{1 , 0 1 8} & \mathbf{2 , 0 0 5} \\ \text { Wester-Silfberg I } & 1 & 1,005 & 0,994 & \mathbf{1 , 9 9 9}\end{array}$




$\begin{array}{lcccc} & F e(C o): & S & A s & (S+A s) \\ \text { Freiberg } & 1 & 1,069 & 0,911 & 1,980 \\ \text { Sala } & 1 & 1,022 & 0,952 & 1,974 \\ \text { Spräkla I } & 1 & 0,985 & 0,984 & 1,969 \\ \text { Spräkla II } & 1 & 0,901 & 1,068 & 1,969\end{array}$

Da gewiss jeder Arsenkies etwas verunreinigt ist und jede Analyse mit Fehlern behaftet sein kann, so düfen wir diesen Varietäten die Formel $\mathrm{Fe}(\mathrm{S}+\mathrm{As})_{2}$ zuschreiben, und annehmen, dass dieselbe Formel auch den Mineralien von Vena und Wester-Silfberg III zukommt, obschon die hier vorkommenden Einschlüsse eine ganz merkliche Abweichung bewirken. Da bei allen vorhergehenden Untersuchungen dieses Minerales die Aufmerksamkeit nicht auf das Vorhandensein von Einschlíssen gerichtet war, so geht daraus nach meiner Ansicht hervor, dass die Formel $\mathrm{Fe}(S, A s)_{2}$ allgemeingultig furdie Arsenkieseist.

Die andere fruher aufgestellte Frage bezieht sich auf die Relation, welche zwischen Krystallstructur und Constitution besteht. Beschränken wir zunächst unsere Untersuchung auf die Eisen-Arsenkiese, so ist es klar, dass, wenn wirklich eine Relation bestebt, diese gleich gut aus jedem Bestandtheile hervorgeht. Denn da $\mathrm{Fe}(\mathrm{S}, \mathrm{As})_{2}$ die Zusammensetzung ausdrückt, so muss, wenn z. B. der Schwefelgehalt vergrössert wird, der Arsengebalt kleiner und der $F e$-Gehalt ein wenig grösser werden. Doch ist es, wie Arzruni vorgeschlagen hat, vortheilhaft, den Schwefel zum Vergleiche zu benutzen, da dieses Element immer als das allerbest bestimmte angesehen werden kann. Denn bei dem Eisen ist eine gewisse Unsicherheit wegen eisenhaltigen Verunreinigungen vorhanden, und bei Arsenik ist die Methode nicht gleich exact wie beim Schwefel.

Bevor ich die hier in Betracht kommenden Zahlen anführe, müssen die Analysenresultate dadurch vergleichbar gemacht werden, dass die bestimmten Verunreinigungen abgezogen werden und der $S$-Gebalt des Ruckstandes berechnet wird. Ich bomerke nochmals zu der folgenden Uebersicht, dass das Mineral von Wester-Silfberg III gewiss wegen des grossen Gehaltes an Einschlüssen eine zu niedrige Zahl geliefert baben muss.

$\begin{array}{lcclc} & S & a & : b: c \\ \text { Spräkla II } & 17,49 & 0,6705: 1: 1,1863 \\ \text { Wester-Silfberg III } & 18,29 & 0,67239: 1: 1,1896 \\ \text { Nyberg } & 19,09 & 0,6792: 1: 1,1910 \\ \text { Spräkla I } & 19,42 & 0,68077: 1: 1,19070 \\ \text { Wester-Silfberg II } & 19,86 & 0,68300: 1: 1,19228 \\ \text { Sala } & 20,39 & 0,68386: 1: 1,2013 \\ \text { Freiberg } & 21,60 & 0,6890: 1: ?\end{array}$


Aus diesen Zahlen geht deutlich hervor, dass der Schwefelgehalt in naher Beziehung zu der Länge der $a$-A xe steht, und wahrscheinlich auch zu der $c-A x e$, denn mit wachsendem Schwefelgehalte vergrössert sich die $a-\mathrm{A} \times \mathrm{x}$ und ebenso die $c$-A x e bei allen Varietäten ausser Nyberg, welches jedoch innerhalb der Beobachtungsfehler liegt. Aus den am meisten extremen Zablen, für Spräikla II und Freiberg, berechnet man, dass jeder Zuwachs der $a$-Axe um 0,00001 einer Vermehrung des Schwefelgehaltes von 0,00222 entspricht, wäbrend Arzruni dies aus seinen Analysen zu 0,00236 berechnete.

Wahrscheinlich ist dieser Zuwachs nicht gleichförmig, sondern er geschiebt in verschiedener Progression ober- und unterhalb des Schwefelgehaltes $49,63 \%$, welcher der Formel $F e S A s$ oder dem Normala rs enkies entspricht. Der Umstand nämlich, dass die Zusammensetzung der Arsenkiese gewiss in allen Fällen wechselt, aber in verhälınissmässig engen Grenzen, kann nur dadurch erklärt werden, dass man die Hauptverbindung dieses Minerals als $F e S A s$ auffasst, welche Verbindung selten ganz rein - wie bei Spräkla I und bei Wester-Silfberg II — vorkommt, sondern gewöhnlich theilweise von den isomorphen Körpern Markasit und Löllingit ersetzt wird. Nach der vorliegenden Untersuchung geht diese Substitution nicht weiter als zu $10 \%$ nach jeder Seite. Will man daher das gegenseitige Verhältniss zwischen krystallographischen Constanten und chemischer Zusammensetzung sludiren, so muss man einerseits in den schwefelreicheren Varietäten eine Substitution von $\mathrm{Fe} \mathrm{S}_{2}$, andererseits bei den schwefelärmeren eine solche von $F e A s_{2}$ voraussetzen. In der That zeigt nämlich die Berechnung, dass man zu einer anderen Relation zwischen der Länge der $a$-Axe und dem Schwefelgehalte kommt, wenn man einerseits von dem Normalarsenkies zu den $S$-reicheren und andererseits zu $S$-ärmeren Varietäten ubergeht, was kaum möglich wäre, wenn die Arsenkiese isomorphe Mischungen von $\mathrm{Fe} \mathrm{S}_{2}$ und $\mathrm{Fe} \mathrm{As}_{2}$ sein würden. Nach der fruther benutzlen, nicht besonders rationellen Ausdrucksweise entspricht also bei höherem $S$-Gehalte jeder Zuwachs der $a$-Axe um 0,00001 einer Vermebrung des $S$-Gehaltes von 0,0026, während bei $S$-ärmeren Arsenkiesen diese Zahl 0,0019 nicht ubersteigt.

Näher auf diesen Gegenstand einzugehen, wurde sebr interessante Gesichtspunkte bieten; wir müssen jedoch davon Abstand nehmen, schon deswegen, weil für solche Auseinandersetzungen genugend exactes Material fehlt. Erinnern wir uns doch, dass unser Mineral kaum ein einziges Mal ganz rein ist, die $S$-Bestimmung also gewöhnlich etwas zu niedrig und auf $0,1 \%$ unsicher sein kann, und dass die Winkel oft bei einzelnen Individuen sehr wechseln. Indessen muss erwähnt werden, dass man aus den Constanten des Normalarsenkieses $a: b=0,682$ und des Markasits 0,752 die entsprechenden Zahlen bei dem Freiberger Mineral zu 0,6876 und des von 
Sala zu 0,6844, anstatt der beobachteten 0,6890 und 0,6839 , berechnet. Bei arsenreicheren Arsenkiesen und Löllingit ist eine derartige Berechnung ganz unstatthaft, bis Analysen und gleichzeilige krystallographische Untersuchung reinen Materials von diesem Minerale vorliegen.

Wie aus der mitgetheilten Tabelle ersichtlich ist, scheint auch die c-Axe mit dem Schwefelgehalte zuzunehmen. Es entsteht daher die Frage, ob vielleicht das Verhältniss $a: c$ constant ist und also eigentlich nur dic $b$-Axe an verschiedenen Varietäten sich ändert.

Bei den gemessenen Arsenkiesen ist dies Verhältniss :
1) Spräkla II
$0,5652 \cdot 1$
2) Wester-Silfberg III
$0,5652: 1$
3) Nyberg
$0,5703: 1$
4) Spräkla I
$0,5717: 1$
5) Wester-Silfberg II $0,5725: 1$
6) Sala
$0,5693: 1$

Da hier auch bei solchen Varietäten, die sehr genaue Messungen zulassen (1, 2, 4 und 5), betrichtliche Abweichungen vorliegen, muss die aufgestellte Frage verneint werden. Eigenthümlich ist jedoch, dass die ganz sicher bestimmten Spräkla II und Wester-Silfberg III einerseits und Spräkla I und Wester-Silfberg II andererseits identische oder fast identische Zahlen gegeben haben. Ob dem Normalarsenkies ( 4 und 5 ) ein gewisses Verhältniss und den mit Löllingit substituirten Arsenkiesen (1, 2 und 3) ein anderes und schliesslich auch den mit Markasit substituirten ein drittes Verhältniss zukommt, scheint hiernach glaublich, muss aber durch Messungen an mehr Varietäten entschieden werden.

Auch die begleitenden Mineralien uben - wie a priori zu erwarten ist - einen gewissen Einfluss auf den auftretenden Typus der Arsenkiese aus, was naturlich am besten hervortritt, wenn man Typen von demselben Fundorte mit einander vergleicht. So treten z. B. die als Spräkla I und Wester-Silfber'g II bezeichneten Varietäten (Normalarsenkiese) beide in körnigem Kalk auf, dieser von Magnetkies, jener von demselben Minerale und Pyrit begleitet. Die als Spräkla II und Wester-Silfberg III bezeichneten, sehr $S$-armen Varietäten dagegen treten in derselben Gebirgsart auf, aber hier fehlen die genannten Lamprite in der unmittelbaren Nähe gänzlich, und das Mineral von Spräkla wird von Magnetit, das von Wester-Silfberg von Magnetit und Zinkblende begleitet. In diesem Falle ist also da ein normaler Arsenkies ausgebildet worden, wo gleichzeitig Magnetkies in reichlicher Menge zugegen ist, während die $S$-ärmeren Varietäten auftreten, wo $S$-reichere Lamprite, wie Eisenkies und Magnetlies, gänzlich fehlen. Bemerkt muss auch werden, dass die $S$-reichste von allen Varietäten, d. i. Freiberg, besonders von viel $\mathrm{Fe} \mathrm{S}_{2}$ als Pyrit begleitet ist. 
Un die isomorphe Vertretung des Eisens durch $N i$ und $C_{0}$ näher zu untersuchen, muss man die kobalthaltigen Varietäten mit reinen Eisenarsenkiesen von ubrigens derselben Constitution vergleichen. Der Arsenkies von Wester-Silfberg, Typus I, unterscheidet sich von Typus II nur dadurch, das von je 33 Eisenatomen eines von Kobalt und Nickel substituirt ist :

\begin{tabular}{|c|c|c|c|c|c|}
\hline \multirow[t]{2}{*}{ t ist: } & \multicolumn{3}{|c|}{ Gehalt von } & \multicolumn{2}{|c|}{ Axenverhalitniss } \\
\hline & $\mathrm{Fe}$ & $(\mathrm{NiCo})$ & $\boldsymbol{S}$ & $a$ & $: b: \quad c$ \\
\hline Wester-Silfberg II & 34,26 & 0 & 19,86 & 0,68300 & $: 1: 1,1923$ \\
\hline - & 33,24 & 1,02 & 19,88 & 0,68407 & $: 1: 1,1910$ \\
\hline
\end{tabular}

Der $\mathrm{C}_{0}$ - und $\mathrm{Ni}$-Gehalt bewirkt also eine geringe Vergrösserung der $a-A x e$, so dass jedes Procent $C o$ und $N i$ die genannte Axe um 0,001 vermehrt. Dagegen wird der Werth der $c$-A xe etwa gleich viel vermindert, was jedoch bei der doppelt so grossen $c$-Axe möglicberweiser innerhalb der Beobachtungsfehler liegt.

Der Arsenkies von Wester-Silfberg III stimmt ziemlich nahe mit dem Vena-Mineral, Typus I, uberein. Ein derartiger Vergleich zeigt :

\begin{tabular}{lccccc} 
& \multicolumn{3}{c}{ Gehalt von } & \multicolumn{2}{c}{ Axenverhältniss } \\
Wester-Silfberg III & 34,66 & 0 & $S$ & $a$ & $: b: c$ \\
Vena I & 34,90 & 2,50 & 18,22 & $0,67239: 1: 1,1896$ & $0,67536: 1: 1,1867$
\end{tabular}

Der $C o$-Gehalt wirkt hier in ganz derselben Weise, wie früher $C o$ und $\mathrm{Ni}$; denn $2 \frac{1}{2} \%$ Co vergrössern die $a$-A xe um 0,0029 ; auch wird hier die $c$-Axe um gleichviel kleiner.

Aus den vorstehenden Untersuchungen geht hervor:

1) Dass der Arsenkies in Zusammensetzung und krystallographisohen Constanten an jedem Fundorte verschiedene Typen zeigt.

2) Dass auch woblausgebildete Krystalle von diesem Minerale oft Beimengungen von anderen Mineralien, wie Magnelit, Magnelkies, Kupferkies, Augit etc. enthalten.

3) Dass die Constitution aller reinen Eisenarsenkiese durch die Formel $\mathrm{Fe}(\mathrm{S}, \mathrm{As})_{2}$ ausgedruckt wird, doch so, dass, wenn man die in der Natur vorkommende Verbindung $\mathrm{FeSAs}$ als Normalarsenkies auffasst, in den Krystallen diese Verbindung von böchstens zebn Procent $\mathrm{Fe}_{2} \mathrm{~S}_{2}$ und der gleichen Menge $\mathrm{Fe} \mathrm{As}_{2}$ substituirt werden kann.

4) Dass eine derartige Substitution in leicht ersichtlicher Weise auf die Krystallstructur zuruckwirkt, sowie auch in gewisser Beziehung zu den begleitenden Mineralien steht.

5) Dass bei den Kobaltarsenkiesen die Substitution des Eisens durch eine geringe Menge $\mathrm{C}_{0}$ und $\mathrm{Ni}$ eine ganz bestimmte gesetzmässige Aenderung der Constanten bewirkt; und dass die Constitution wahrscheinlich durch die Formel $(\mathrm{Fe}, \mathrm{CoN} i)(\mathrm{As}, \mathrm{S})_{2}$ ausgedrückt wird. 